\title{
Diabetic and non-diabetic human cornea and tear $\gamma$-glutamyl transpeptidase activity
}

This article was published in the following Dove Press journal:

Clinical Ophthalmology

9 January 2013

Number of times this article has been viewed

\author{
Jordan M Burnham' \\ Monali Sakhalkar' \\ Marlyn P Langford' \\ Chanping Liang' \\ Thomas B Redens' \\ Sushil K Jain ${ }^{2}$ \\ 'Department of Ophthalmology, \\ ${ }^{2}$ Department of Pediatrics, Louisiana \\ State University Health Sciences \\ Center, Shreveport, LA, USA
}

Correspondence: Marlyn P Langford Department of Ophthalmology, Louisiana State University Health Sciences Center, I50 I Kings Hwy, PO Box 33932

Shreveport, LA 7II30-3932, USA

Tel +I 31867550I8

Email mlangf@Isuhsc.edu
Background: Diabetes-related eye disease is due in part to oxidative stress. Gamma-glutamyl transpeptidase (GGT) is a $\gamma$-glutamyl cycle enzyme that protects against oxidative stress via glutathione recapture. This study investigates corneal and Schirmer tears GGT activity in diabetic and non-diabetic adults aged 50 to 83 years old.

Methods: GGT activity was determined by colorimetric assay on 50 corneas from 14 diabetic (without keratopathy) and 20 non-diabetic donors and on Schirmer type 1 test strips (no anesthesia) of 14 diabetic and 14 non-diabetic subjects.

Results: Type 1 (T1) diabetic cornea GGT activity was 40\% lower than Type 2 (T2) diabetic cornea GGT activity $(P=0.04)$, but GGT activity was similar for corneas (without keratopathy) from diabetic and non-diabetic donors ( $P \geq 0.44$ for all). The number of endothelial cells/unit of GGT activity in diabetic corneas was $22 \%$ higher $(P=0.1)$ than in non-diabetic corneas. GGT activity per Schirmer strip and GGT activity per mm of tears were $36 \%$ and $50 \%$ higher ( $P \leq 0.008$ for all) for non-diabetic (tear volume dependent) than diabetic donors (tear volume independent), respectively. GGT activity per mm was 50\% lower in $\mathrm{T} 1$ than $\mathrm{T} 2$ diabetics $(P=0.02)$. Higher tear GGT activity in non-diabetic than diabetic females $(P \leq 0.05)$ was due to higher GGT activity in the African American females.

Conclusion: GGT activity was less in T1 than T2 diabetics, but comparable to non-diabetic corneas. Schirmer tear GGT activity in diabetic eyes was tear volume independent, less in T1 than T2, lower than in tear volume dependent, non-diabetic female eyes. Low cornea and tear GGT activity suggests loss of antioxidant potential and supports ocular antioxidant therapy for diabetic patients.

Keywords: cornea, diabetes, endothelium, epithelium, eye, $\gamma$-glutamyl transpeptidase, tear, oxidative stress

\section{Introduction}

Gamma-glutamyl transpeptidase (GGT) activity plays a key role in protecting cells against oxidative stress, via recapture of extracellular glutathione (GSH; a potent intracellular antioxidant), and xenobiotic detoxification. ${ }^{1-4}$ The physiological functions and importance of GGT in human ocular tissues have not been elucidated fully, but elevated plasma GGT and diabetes are risk factors for senile cataract formation. ${ }^{5}$ In addition, age and diabetes-associated losses of lens and retinal GGT and GSH precede lens intumescences and opacification and retinal dysfunction, respectively. ${ }^{6-10}$ GGT expression by quiescent endothelial cells lining the posterior corneal surface, metabolically active columnar epithelial cells along the anterior stromal surface, and senescent surface epithelial cells at the tear/corneal interface ${ }^{11}$ supports cell-specific protective effects of corneal GGT against metabolic and environmental oxidative stress 
factors. Notably, the physiological importance of intra-ocular GGT is supported by the report that inhibition of the corneal endothelial cell GGT activity increases corneal thickness (hydration) and decreases transparency. ${ }^{12}$

Diabetes and age-related corneal and lens pathologies have been attributed in part to oxidative stress associated with anaerobic metabolism and exposure to the external environment. ${ }^{13,14}$ Diabetes-associated corneal complications include decreased corneal sensitivity (neuropathy), dryness and thickness, reduced barrier function, and changes in endothelial cell morphology and density. ${ }^{15-23}$ Diabetic keratopathy (characterized by corneal thinning, disorganization, and persistent epithelial defects) occurs commonly in subjects with longstanding, poorly controlled diabetic disease. ${ }^{23}$ Moreover, advanced glycation end products (associated with inactivation of GSH and GSHrelated enzymes) are increased in the corneas of subjects with diabetes and proliferative diabetic retinopathy suggesting glycation due to loss of antioxidant potential may play a role in diabetic keratopathy. ${ }^{24}$ Age-related corneal pathologies include changes in thickness, elasticity, endothelial cell morphology, and density. ${ }^{25-27}$

Corneal endothelial cell GGT activity decreases with age ${ }^{28}$ and reflex tear GGT activity is higher in older individuals with refraction defects. ${ }^{29}$ It is not known if diabetes affects GGT activity in human corneas and tears. The present study investigates the levels of GGT activity in corneas and tears of adult diabetic and non-diabetic subjects. The results suggest that (1) corneal GGT activity levels in age-matched non-diabetic and diabetic eyes without keratopathy are essentially the same, (2) GGT activity per endothelial cell in type 1 (T1) diabetic cornea is lower than in type 2 (T2) diabetic corneas, and (3) Schirmer tear GGT activity is tear volume independent in diabetic eyes and higher in tear volume dependent non-diabetic eyes.

\section{Material and methods Human corneas}

Fifty corneas from 34 donors $\geq 50$ years old were provided through the auspices of the Northwest Louisiana Eye Bank, Shreveport, LA and the Southern Eye Bank, New Orleans, LA. Thirty-one corneas were from 20 non-diabetic donors (eleven pairs and nine singles). ${ }^{28}$ Nineteen corneas were from 14 (nine T1 and five T2) diabetic donors (six pairs and seven singles). The donor globes and/or corneal buttons were obtained less than 8 hours post mortem, frozen in corneal transport media, and transported on ice. The cause of death (COD), limited past medical history, age, and the corneal endothelial cell densities (cells $/ \mathrm{mm}^{2}$ ) were provided for most donors. The globes and corneas were thawed quickly to $37^{\circ} \mathrm{C}$ and corneal buttons were produced using $11.0 \mathrm{~mm}$ trephines (Hampton Medical Devices, LLC, Valley Park, MO). The corneal buttons were gently rinsed three times with $1 \mathrm{~mL}$ phosphate buffered saline (PBS, $\mathrm{pH}$ 7.2) to remove the transport medium. The corneas were assayed for GGT as described below and then rinsed with PBS to remove GGT medium. The anterior epithelial and posterior endothelial layers were separated by a dissection of the stroma using iris forceps, surgical blades and binocular 10X magnification. Corneal epithelium and endothelium sections were stored at $4^{\circ} \mathrm{C}$ in PBS until assayed for GGT activity (note: only corneas, endothelial, and epithelial sections with intact endothelial and epithelial were assayed).

\section{Schirmer test strip tears}

Twenty-eight bilateral Schirmer type 1 (without topical anesthesia) tests were performed on consenting individuals during referral or routine visits to the Department of Ophthalmology Eye Clinic at the Louisiana State University Health Sciences Center in Shreveport, LA. Schirmer tests were performed as per the manufacturer's instructions (HaagStreit, UK Ltd, Harlow, UK). ${ }^{30}$ Briefly, the Schirmer test strips were applied to unanesthetized eyes of consenting donors for 5 minutes, the strips were removed, the mm of tear fluid per test strip was measured and recorded, the test strips were air-dried, sealed in labeled air-tight plastic bags, and stored at $-10^{\circ} \mathrm{C}$ for up to 2 weeks (to prevent GGT degradation). ${ }^{31}$ All subjects with keratitis/conjunctivitis, taking ocular or renal medications, or failing to produce $10 \mathrm{~mm}$ of tears in 5 minutes were excluded.

\section{Corneal and tear GGT activity}

Corneal and Schirmer tear GGT activity was determined using standard assay methods as described previously. ${ }^{11,32}$ Briefly, whole cornea, endothelial, and epithelial sections were placed in GGT assay medium and incubated at room temperature for 3 hours. The units of GGT activity (ie, nanomoles of para-nitroanalide converted per hour) were determined by colorimetric analysis. The estimated number of endothelial cells/unit of GGT activity for each intact endothelial section was calculated by dividing the total number of endothelial cells per $11.0 \mathrm{~mm}$ diameter endothelial corneal button (ie, average number of endothelial cells $/ \mathrm{mm}^{2}$ (density) multiplied by the endothelial surface area) by the units (U) of endothelial GGT activity. The Schirmer test tear/conjunctival GGT activity was assayed by placing the strip in $1 \mathrm{~mL}$ of GGT 
assay medium and incubating for 3 hours at room temperature. The mean tear GGT activity (U/strip and U/mm) per donor was determined from bilateral tear strips.

\section{Immunohistochemistry}

Immunoreactive GGT was detected in epithelial cells of cryo-sectioned conjunctival tissue from a non-diabetic human cadaver eye following reaction with a 1:100 dilution of rabbit antibody to GGT (anti- $\gamma$-glutamyl transferase, \#53506; AnaSpec Inc, Freemont, CA) and DAPI nuclear stain using previously reported methods. ${ }^{11,28}$ Digitalized images were captured with a CoolSNAP fx monochrome CCD camera (Photometrics, Tucson, AZ) and color images produced with Scanalytics IPLab software (Spectra Services Inc, Ontario, NY).

\section{Statistical analysis}

Mean GGT activity per corneal donor was calculated based upon the GGT per corneal pair. Tear per conjunctival GGT activity per subject was calculated based upon the mean GGT activity of the bilateral Schirmer test strips (note: no significant differences were detected between the left and right corneas or tears samples by paired $t$-test analysis.) Group means and statistical inferences between groups were determined utilizing Mann-Whitney rank sum tests and linear regression analyses using Microsoft Excel (Richmond, VA) and SigmaPlot software (Systat Software, San Jose, CA). An unpaired Student's $t$-test was used when the data did not meet Mann-Whitney test criteria. Mean differences were considered to be statistically significance at $P \leq 0.05$.

\section{Results}

\section{Corneal and tear donors}

The mean age ( \pm standard deviation; SD) of the 14 diabetic corneal donors ( $63 \pm 8$ years; range from 50 to 83 years) was essentially identical to the mean age of the 20 non-diabetic corneal donors (63 \pm 10 years; range from 51 to 83 years).

COD for diabetic donors
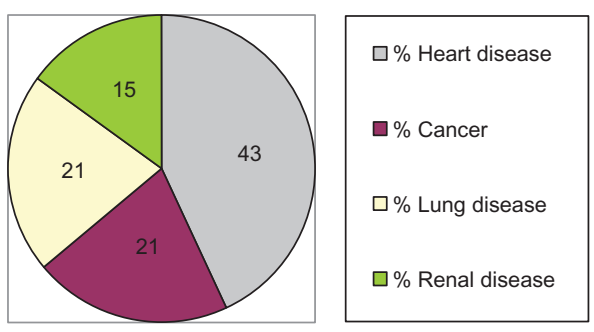

The COD for the diabetic corneal donors included heart disease ( $43 \%$; six of 14$)$, cancer $(21 \%$; three of 14$)$, lung disease (21\%; three of 14$)$ and renal disease (15\%; two of 14$)$ (Figure 1). In comparison, the COD for non-diabetic control corneal donors included heart disease (45\%; nine of 20 ), cancer (20\%; four of 20$)$, lung disease (20\%; four of 20$)$, and miscellaneous ( $15 \%$; three of 20$)$. The results suggest that the non-diabetic and diabetic donor groups were similar with respect to mean age and COD. The cornea donors' gender and race were not provided.

Fourteen diabetic [(mean age $61 \pm 9.7$ years); six Caucasian females, four African-American females, three Caucasian males and one African-American male] and 14 non-diabetic Eye Clinic subjects [(mean age $67 \pm 9.8$ years, $P=0.1$ ); four Caucasian females, seven African-American females, two Caucasian males and one African-American male] consented to provide bilateral Schirmer test strip tear samples for analysis. Notably, nine (64\%) of the tear donors had T1 diabetes and five (36\%) had T2 diabetes (Figure 2). Diabetic retinopathy was reported in $36 \%$ (five of 14) of the diabetic tear donors [three T1 (33\%) and two (40\%) T2 diabetic donors]. In contrast, seven $(50 \%)$ of the adult non-diabetic control patient tear donors were pre-op cataract cases, five (36\%) were refraction cases and two (14\%) had miscellaneous non-medicated, non-dry-eye, non-glaucomatous, and noninflammatory ocular symptoms.

\section{GGT activity in whole cornea, endothelia, and epithelia sections}

The GGT activity in whole corneas from non-diabetic control donors $(65 \pm 35 \mathrm{U})$ was slightly higher than in the whole corneas of diabetic donors ( $56 \pm 30 \mathrm{U} ; P=0.45$ ) (Figure 3A). The GGT activity for all non-diabetic donor whole corneas $(62.4 \pm 36 \mathrm{U} ; \mathrm{n}=31)$ was not significantly different from the GGT activity for all diabetic donor whole corneas ( $56 \pm 31 \mathrm{U}$; $\mathrm{n}=19 ; P=0.53$ ). The mean ( \pm standard error of the mean; SEM) GGT activity for 16 non-diabetic donor endothelial section

COD for non-diabetic donors

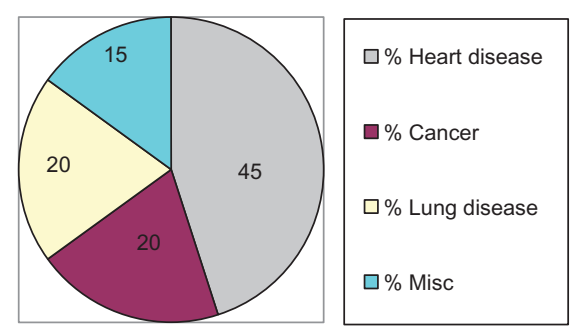

Figure I COD for the diabetic and non-diabetic corneal donors. Abbreviation: COD, cause of death. 
Diabetic tear donors
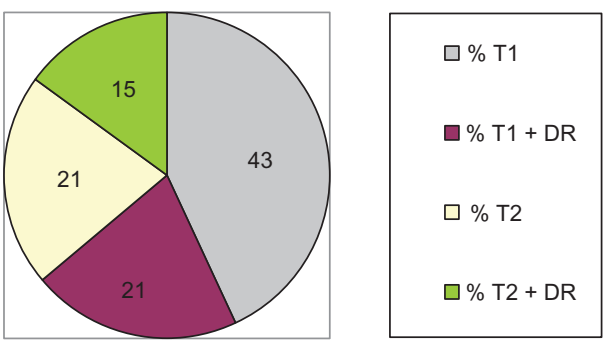

Figure 2 Clinical evaluation of diabetic and non-diabetic tear donors.

Abbreviations: TI, type I diabetes; T2, type 2 diabetes; DR, diabetic retinopathy.

pairs $(29 \pm 11 \mathrm{U})$ was similar to that for eleven diabetic donor endothelial section pairs $(25 \pm 8 \mathrm{U} ; P=0.21)$. The levels of corneal epithelial GGT activity in 19 non-diabetic cornea donors $(26 \pm 18 \mathrm{U})$ were identical to that for 10 diabetic cornea donors ( $26 \pm 18 \mathrm{U} ; P=0.99)$. The GGT activity in nine T1 diabetic corneas $(48 \pm 25 \mathrm{U})$ was significantly lower than in five $\mathrm{T} 2$ corneas (79 $\pm 36 \mathrm{U} ; P=0.04$ ), and approached significance versus the non-diabetic corneas $(P=0.13)$ (Figure 3B). The results show that corneal GGT activity in the diabetic eyes (without keratopathy) and non-diabetic eyes are comparable, and suggest GGT activity is lower in $\mathrm{T} 1$ than $\mathrm{T} 2$ diabetic corneas.

\section{Endothelial cell density and GGT activity}

The following analyses were performed to ascertain the GGT activity per corneal endothelial cell in adult non-diabetic and diabetic donor corneas. The mean cell density $( \pm \mathrm{SEM})$ of intact corneal endothelial sections for the non-diabetic donor corneas (2644 \pm 195 cells $/ \mathrm{mm}^{2}$ ) was not significantly different than the average cell density of the diabetic (predominantly T1) donor corneas $\left(2,581 \pm 172\right.$ cells $\left./ \mathrm{mm}^{2} ; P=0.68\right)$ (Figure 4A).
Non-diabetic tear donors

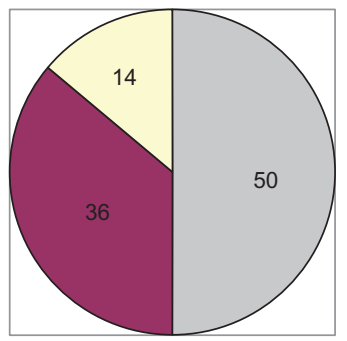

$\square \%$ Cataract

๑ $\%$ Refraction

$\square \%$ Misc

However, the mean estimated number of endothelial cells per unit of GGT activity was $22 \%$ lower in non-diabetic $(10,630 \pm 4335$ endothelial cells/unit) than in the diabetic corneas $(13,566 \pm 5494$ endothelial cells/unit: $P=0.13$; Mann-Whitney) (Figure 4B). The results suggest slightly lower GGT activity in diabetic cornea endothelial cells.

\section{Effect of age, race, gender, and tear volume on Schirmer GGT activity}

Bilateral Schirmer tear production for 28 eye clinic donors was measured and the Schirmer test strips assayed for GGT activity [no differences were detected between left and right eyes $(P=0.95)]$. No differences were detected for tear production with respect to donor race or gender. Scatter plot and linear regression analyses suggest a decline in the non-diabetic and diabetic Schirmer strips GGT activity levels with donor age (left eye; $\mathrm{R}=0.177, t=11.4$, slope $=-0.128$ and right eye; $\mathrm{R}=0.247, t=11.1$, slope $=-0.164)$ (Figure 5A); consistent with an aged-related decrease previously observed for Schirmer tear production by hospital outpatients. ${ }^{30}$ More interestingly, the

A

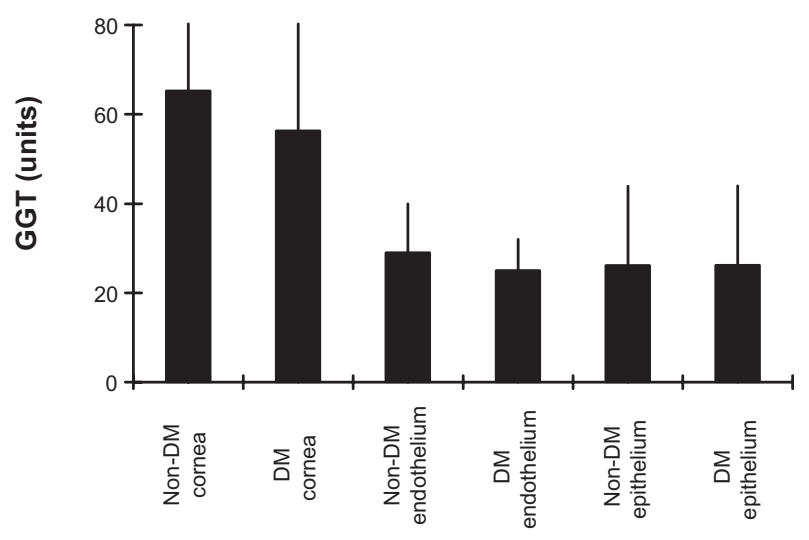

B

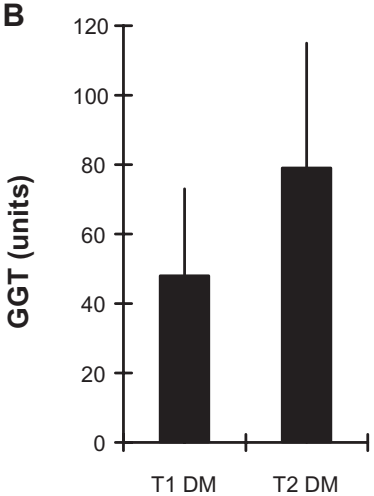

Figure 3 Non-diabetic and diabetic corneal GGT activity. (A) Mean \pm SEM for corneal, endothelial and epithelial GGT activity within non-DM and DM donor groups. (B) Comparison of GGT activity in TI and T2 diabetic corneas.

Abbreviations: GGT, $\gamma$-glutamyl transpeptidase; SEM, standard error of the mean; DM, diabetes mellitus; TI, type I diabetes; T2, type 2 diabetes. 

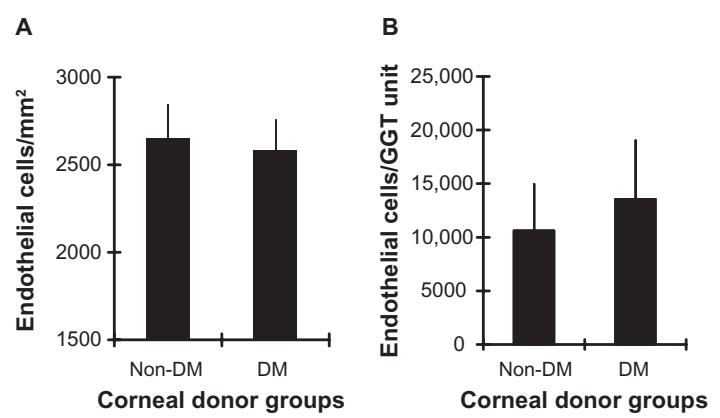

Figure 4 Endothelial cell density and GGT activity. (A) Mean ( \pm SEM) cornea endothelial cell density in 50 to 83 year old non-diabetic and/or diabetic donor groups. (B) Mean ( \pm SEM) number of corneal endothelial cells/unit of GGT activity in the non-diabetic and/or diabetic donor groups.

Abbreviations: GGT, $\gamma$-glutamyl transpeptidase; SEM, standard error of the mean; DM, diabetes mellitus.

GGT activity per Schirmer strip from non-diabetic donors was closely associated with the tear volume ( $\mathrm{mm}$ of tears produced per 5 minutes) (slope $=16.44 ; \mathrm{R}=0.528, t=2.45, P=0.02$ ) (Figure 5B). That is, the greater the tear volume per strip the higher the GGT activity. In contrast, diabetic tear GGT activity was independent of tear volume (slope $=1.37 ; \mathrm{R}=0.23$, $t=0.821, P=0.428$ ); suggesting a non-aqueous source for GGT. In this regard, it should be noted that forniceal conjunctiva cells express immunoreactive GGT (Figure 5C), human conjunctival epithelial cells exhibit GGT activity, ${ }^{33}$ and GGT activity was localized predominantly to the area of the Schirmer strip placed in the conjunctival sac (Figure 5D). Taken together, these results support greater tear and conjunctiva GGT activity in non-diabetic than diabetic eyes, independence of Schirmer GGT activity and tear volume in diabetic eyes, dependence of Schirmer GGT activity on tear volume in non-diabetic eyes, and provide evidence consistent with the idea that Schirmer GGT activity is comprised of free and membrane-associated GGT.

\section{Diabetic and non-diabetic Schirmer tear production and GGT activity}

Schirmer type 1 tear production ( \pm SEM) by the 14 diabetic donors (22.6 $\pm 8 \mathrm{~mm} / 5$ minutes; 10 females and 4 males) was
A

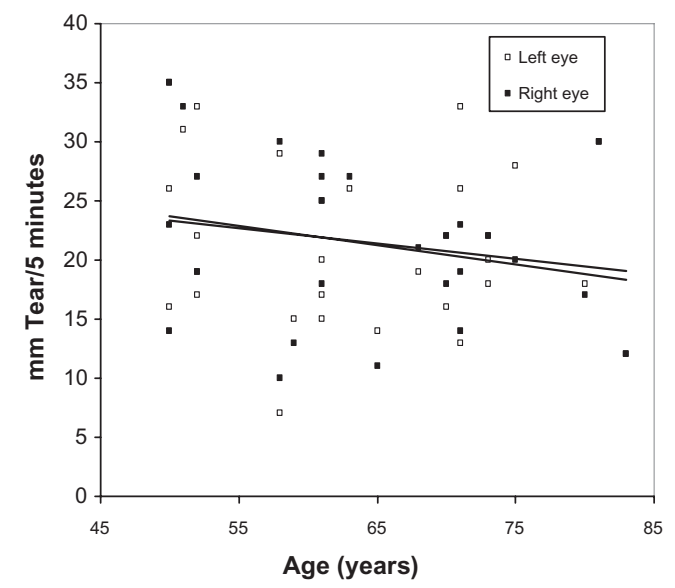

C

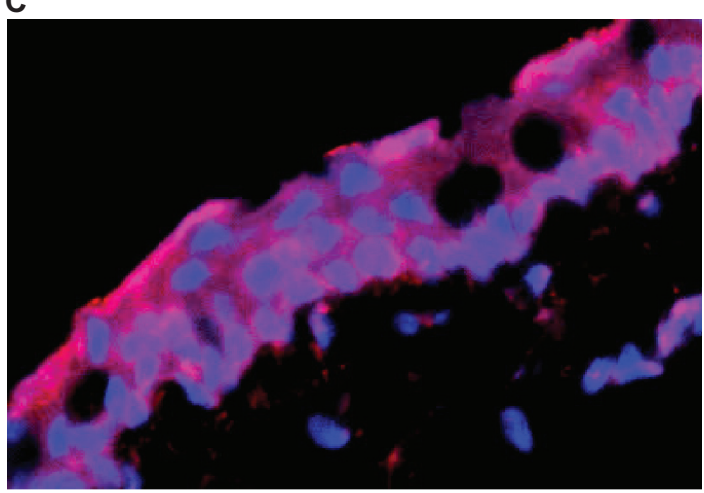

B

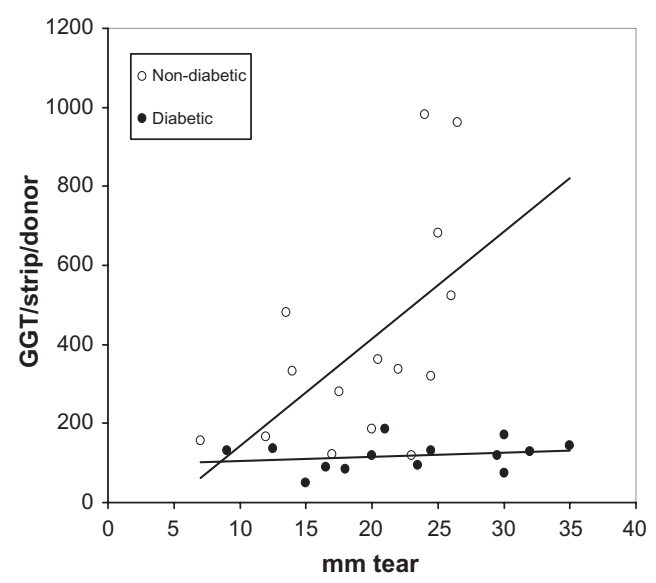

D

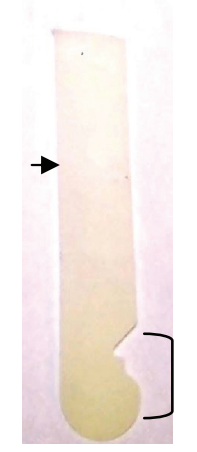

Figure 5 (A) Scatter graph plot of the bilateral Schirmer tear production of all adult donors versus age showing a general decline in tear production (left and right trend lines) with donor age. (B) Scatter graph plot with trend line (linear regression) of tear volume (mm) per Schirmer test strip versus GGT activity per Schirmer test strip for non-diabetic and diabetic tear donors. (C) Distribution of GGT (red) on DAPI-stained (blue nuclei) human conjunctival epithelial cells ( $\times 400)$. (D) GGT reaction substrate (yellow color) was concentrated on the Schirmer's test strip in the conjunctival contact area (bracket) and decreased toward the tear meniscus (arrow). Abbreviation: GGT, $\gamma$-glutamyl transpeptidase. 
not significantly different from that of the 14 non-diabetic donors $(20.4 \pm 4.9 \mathrm{~mm} / 5$ minutes; 11 females and 3 males; $P=0.39$ ) (Table 1). The Schirmer GGT activity for diabetic donors was 3.5-fold lower than for non-diabetic donors $(118 \pm 38 \mathrm{U} /$ strip versus $418 \pm 283 \mathrm{U} /$ strip; $P=0.0006)$ Similarly, the GGT activity per mm of tear volume was 1.9-fold lower in diabetic than non-diabetic donors (10.5 $\pm 6.1 \mathrm{U} / \mathrm{mm}$ versus $20.4 \pm 11.4 \mathrm{U} / \mathrm{mm} ; P=0.008)$. Interestingly, the Schirmer GGT activity of diabetic females was lower than for non-diabetic females $(202 \pm 79 \mathrm{U} / \mathrm{strip}$ versus $447 \pm 294 \mathrm{U} /$ strip; $P=0.014$ ). Concomitantly, the GGT activity per mm of tear was significantly lower in the diabetic than non-diabetic females $(8.4 \pm 2.8 \mathrm{U} / \mathrm{mm}$ versus $22.3 \pm 12.2 \mathrm{U} / \mathrm{mm} ; P=0.002)$. Further, GGT activity per strip and GGT activity per mm of tear were significantly lower in diabetic Caucasian than in non-diabetic African-American females $(201 \pm 97$ versus $465 \pm 285 \mathrm{U} /$ strip; $P=0.05$ and $8.3 \pm 2.5$ versus $23.6 \pm 12.7 \mathrm{U} / \mathrm{mm} ; P=0.01$ ). [No differences in tear production were detected $(P=0.24)$.] No other differences in Schirmer GGT activity were detected between diabetic and non-diabetic females and males. No significant differences were detected between $\mathrm{T} 1$ and $\mathrm{T} 2$ diabetic donors with respect to tear production $(P=0.19)$ or GGT activity per strip $(P=0.36)$, but GGT activity per mm of tear volume was significantly lower in the T1 than $\mathrm{T} 2$ diabetic donors $(7.7 \pm 2.6$ versus $15.4 \pm 7.8 \mathrm{U} / \mathrm{mm} ; P=0.02)$. These results support lower tear and conjunctival GGT activity in diabetic than non-diabetic adults, particularly between diabetic Caucasian versus non-diabetic African-American females, and suggest differences in GGT activity per mm of Schirmer tear between T1 and T2 diabetic eyes.

\section{Discussion}

The relative levels of cornea and tear GGT activity in 50 to 83 year-old diabetic and non-diabetic adults are presented. Comparable levels of GGT activity were detected in diabetic corneas without keratopathy as in non-diabetic corneas. Accordingly, no significant differences were detected in endothelial and epithelial GGT activity and endothelial cell densities for these donor cornea groups ( $P \geq 0.4$ for all). However, the number of endothelial cells/unit of GGT activity was $20 \%$ higher in the diabetic than non-diabetic donor corneas; suggesting that diabetic corneal GGT expression per endothelial cell is reduced. The lower GGT expression per corneal endothelial cell is similar to the reported reduction in endothelium GGT in the retinal vessels of animals with diabetic retinopathy. ${ }^{9}$ Also, $\mathrm{T} 1$ diabetic corneal GGT activity was $40 \%$ lower than in the T2 diabetic donor corneas, consistent with the concept that $\mathrm{T} 1$ diabetic cornea may be more susceptible to metabolic and environmental stress than T2 diabetic corneas. ${ }^{20,34}$ Thus, the putative decrease in endothelial cell GGT expression may represent an early pathological event in diabetic cornea as observed in precataractous cortical lens epithelial cells. ${ }^{6-8}$

The analyses of the GGT activity on Schirmer type 1 test for the 14 non-diabetic and 14 diabetic Eye Clinic tear donors indicated GGT activity was significantly higher in non-diabetic eyes than in diabetic eyes per Schirmer strip and

Table I GGT activity in tear samples from DM and N-DM male and female adults

\begin{tabular}{|c|c|c|c|c|c|c|}
\hline Patient groups & $\begin{array}{l}\text { Females } \\
(n=)\end{array}$ & $\begin{array}{l}\text { Males } \\
(n=)\end{array}$ & Age \pm SD & $\begin{array}{l}* \text { Tear production } \\
\pm \text { SEM }\end{array}$ & $\begin{array}{l}\text { GGT/strip } \\
\pm \text { SEM }\end{array}$ & $\begin{array}{l}\text { GGT/mm } \\
\pm \text { SEM }\end{array}$ \\
\hline N-DM & 11 & 3 & $67 \pm 10$ & $20.4 \pm 4.9$ & $418 \pm 283$ & $20.4 \pm 11.4$ \\
\hline DM & 10 & 4 & $61 \pm 10$ & $22.6 \pm 8.0$ & $118 \pm 38$ & $10.5 \pm 6.1$ \\
\hline$P$ & & & 0.1 & 0.39 & 0.0006 & 0.008 \\
\hline N-DM females & 11 & & $66 \pm 8.5$ & $21.2 \pm 4.8$ & $447 \pm 294$ & $22.3 \pm 12.2$ \\
\hline DM females & 10 & & $61 \pm 11$ & $24.9 \pm 6.6$ & $202 \pm 79$ & $8.4 \pm 2.8$ \\
\hline$P$ & & & 0.22 & 0.15 & 0.014 & 0.002 \\
\hline N-DM males & & 3 & $69 \pm 16$ & $17.5 \pm 4.8$ & $237 \pm 109$ & $13.6 \pm 4.3$ \\
\hline DM males & & 4 & $61 \pm 3$ & $16.8 \pm 9.1$ & $202 \pm 43$ & $15.6 \pm 9.5$ \\
\hline$P$ & & & 0.31 & 0.90 & 0.56 & 0.74 \\
\hline N-DM black females & 7 & & $65.0 \pm 9$ & $20.0 \pm 5.0$ & $465 \pm 285$ & $23.6 \pm 12.7$ \\
\hline DM white females & 6 & & $60.8 \pm 12$ & $24.3 \pm 7.4$ & $20 I \pm 97$ & $8.3 \pm 2.5$ \\
\hline$P$ & & & 0.48 & 0.24 & 0.05 & 0.01 \\
\hline T2 DM & 3 & 2 & $65 \pm 11$ & $18.7 \pm 8.7$ & $|3| \pm 3 \mid$ & $15.4 \pm 7.8$ \\
\hline TI DM & 7 & 2 & $58 \pm 8$ & $24.7 \pm 7.1$ & $|I| \pm 4 \mid$ & $7.7 \pm 2.6$ \\
\hline$P$ & & & 0.19 & 0.19 & 0.36 & 0.02 \\
\hline
\end{tabular}

Notes: *Tear production (mm per 5 minutes) was measured by Schirmer tests.

Abbreviations: GGT, $\gamma$-glutamyl transpeptidase; DM, diabetic; N-DM, non-diabetic; SEM, standard error of the mean; TI, type I diabetes; T2, type 2 diabetes. 
per mm of tear measured $(P \leq 0.008)$. The higher Schirmer GGT activity of the non-diabetic subjects was due to higher levels of tear GGT activity in non-diabetic than diabetic females. No differences were observed between non-diabetic and diabetic males. Previous investigators have reported a positive correlation between GGT activity in onion-induced reflex tears of women and their age. ${ }^{29}$ Schirmer GGT activity in non-diabetic and diabetic Caucasian or African-American females were not different (not shown), but non-diabetic adult African-American females had higher tear GGT activity than diabetic Caucasian females. The apparent race difference between females was likely due to the 2-fold greater GGT activity per strip $(P=0.05)$ observed in three Caucasian and three African-American non-diabetic females examined for refraction defects than in the Caucasian and four African-American pre-op females. The observed association between refraction defects and elevated tear Schirmer GGT activity was consistent with a previous report of elevated GGT activity in reflex tears of females ( $\geq 40$ years old) with refraction defects. ${ }^{29}$

Why lower levels of Schirmer GGT activity were detected in the diabetic eyes is unknown. The finding that diabetic donor Schirmer GGT activity was independent of tear volume, but non-diabetic donor Schirmer GGT activity was closely associated with tear volume suggests that GGT in the tear fluid is reduced in diabetic eyes. The likelihood that most of the tear per conjunctival GGT activity adsorbed on each Schirmer test strip is derived from GGTpositive conjunctival epithelial cells and/or leukocytes ${ }^{33,35}$ is supported by the physical contact between the Schirmer test strip and the conjunctiva and the localization of GGT activity to the area of the Schirmer strip that comes in contact with the conjunctiva. Thus, the basal level of GGT on each Schirmer strip likely represents the adsorption of GGT-positive conjunctival cells, extruded leukocytes, and/or debris. Concomitantly, the observed volume dependency for GGT activity in non-diabetic eyes was likely due to release of GGT from cells of conjunctiva and/or associated exocrine tissue into the tear fluid, but other factors cannot be ruled out. Further, T1 diabetic donor GGT activity per mm of Schirmer tear was significantly lower than in the T2 diabetic donors $(P=0.02)$ suggesting that the diabetes-associated defect is more pronounced in $\mathrm{T} 1$ diabetes. Lower GGT activity in diabetic tear per conjunctival tissue adsorbed to the Schirmer test strip is consistent with the results of previous studies showing that GGT levels are reduced in ocular tissue of diabetic individuals. ${ }^{6-10}$ These findings are consistent with the suggestion that diabetes-associated oxidative stress plays a role in corneal and conjunctival pathologies. Accordingly, loss of GGT suggests loss of GSH recapture potential, depletion of $\mathrm{GSH}$, reduction in protection against oxidative stress, and increased corneal and conjunctival cell morbidity. Additional studies are needed to clearly define the effects of diabetes mellitus on GGT expression and release from conjunctival and lacrimal cells.

Several studies have reported dry eye in diabetic children and adults. ${ }^{16,17,36,37}$ However, Schirmer type 1 measurements for the non-diabetic $(20.4 \pm 4.9 \mathrm{~mm})$ and diabetic $(22.6 \pm 8 \mathrm{~mm})$ donors were not significantly different. The failure to detect differences in tear production in our study may be due to differences in experimental protocols (ie, analysis of onion-induced reflex tears, Schirmer tests performed on unanesthetized eyes, and anesthetized eyes) or patient populations (ie, exclusion criteria, diabetic subjects with diabetic retinopathy, or T1 versus T2 diabetic eyes). Notably, tear production by our donors was within the normal ranges reported previously $(18 \pm 5 \mathrm{~mm}$ to $22.98 \pm 1.05 \mathrm{~mm}) .^{37,38}$

In summary, corneal GGT activity in diabetic adult eyes without keratopathy was shown to be very similar to that in non-diabetic adult eyes, but loss of endothelial cell GGT activity in diabetic corneas appears to be an early complication. Schirmer tear GGT activity was lower in diabetic than non-diabetic eyes and appeared to be associated with less GGT in the tear fluid. The possibility that exclusion criteria, duration and severity of each donor's diabetes, and systemic disease, ${ }^{21,23}$ may have an effect on corneal GGT and our results cannot be ruled out. Additional studies are needed; to determine the prognostic value of Schirmer tear GGT activity in assessing extra-ocular complications of diabetes, to investigate GGT activity in cornea and conjunctiva of eyes with diabetic keratopathy, and to support therapeutic approaches for management of diabetes-associated changes in extraocular tissue due to oxidative stress. $^{13,14,39,40}$

\section{Acknowledgments}

The authors thank Christopher Duggan for excellent technical support. The authors gratefully acknowledge the Northwest Louisiana Eye Bank, Shreveport, LA and the Southern Eye Bank of New Orleans, LA for providing the corneal tissue utilized in this investigation. The authors thank the Medical Student Research Program, Louisiana State University Health Center-Shreveport (JB), and the Department of Ophthalmology Resident Research Program, LSU Health, Shreveport, LA (MS) for their support. These investigations 
were performed in accordance with the tenets of the 1989 Declaration of Helsinki and in accordance with protocols approved by the Louisiana State University Health Sciences Center Internal Review Board.

\section{Disclosure}

The study was supported in part by the Medical Student Research Program, Louisiana State University Health CenterShreveport (JB) and the Department of Ophthalmology Resident Research Program, LSU Health, Shreveport, LA (MS). The authors have no conflicts of interest with regard to this work.

\section{References}

1. Griffith OW, Meister A. Glutathione: interorgan translocation, turnover, and metabolism. Proc Natl Acad Sci U S A. 1979;76(11): 5606-5610.

2. Cotgreave IA, Schuppe-Koistinen I. A role for gamma-glutamyl transpeptidase in the transport of cystine into human endothelial cells: relationship to intracellular glutathione. Biochim Biophys Acta. 1994;1222(3):375-382.

3. Sweiry JH, Sastre J, Viña J, Elsässer HP, Mann GE. A role for gamma-glutamyl transpeptidase and the amino acid transport system xc- in cystine transport by a human pancreatic duct cell line. J Physiol. 1995;485 (Pt 1):167-177.

4. Miller SP, Arya DV, Srivastava SK. Studies of gamma-glutamyl transpeptidase in human ocular tissues. Exp Eye Res. 1976;22(4): 329-334.

5. Donnelly CA, Seth J, Clayton RM, Phillips CI, Cuthbert J, Prescott RJ. Some blood plasma constituents correlate with human cataract. $\mathrm{Br} J$ Ophthalmol. 1995;79(11):1036-1041.

6. Rathbun WB, Schmidt AJ, Holleschau AM. Activity loss of glutathione synthesis enzymes associated with human subcapsular cataract. Invest Ophthalmol Vis Sci. 1993;34(6):2049-2054.

7. Sadasivudu B, Rao GN, Swamy M. Gamma-glutamyl transpeptidase and gamma-glutamyl cysteine synthetase activity in human lens tissue. IRCS Med Sci. 1981;9:55-56.

8. Chévez-Barrios P, Wiseman AL, Rojas E, Ou CN, Lieberman MW. Cataract development in gamma-glutamyl transpeptidase-deficient mice. Exp Eye Res. 2000;71(6):575-582.

9. Kowluru R, Kern TS, Engerman RL. Abnormalities of retinal metabolism in diabetes or galactosemia. II. Comparison of gammaglutamyl transpeptidase in retina and cerebral cortex, and effects of antioxidant therapy. Curr Eye Res. 1994;13(12):891-896.

10. Kern TS, Kowluru RA, Engerman RL. Abnormalities of retinal metabolism in diabetes or galactosemia: ATPases and glutathione. Invest Ophthalmol Vis Sci. 1994;35(7):2962-2967.

11. Langford MP, Redmond P, Chanis R, Misra RP, Redens TB. Glutamate, excitatory amino acid transporters, $\mathrm{Xc}$ - antiporter, glutamine synthetase, and gamma-glutamyl transpeptidase in human corneal epithelium. Curr Eye Res. 2010;35(3): 202-211.

12. Anderson EI, Wright DD. The roles of glutathione reductase and gammaglutamyl transpeptidase in corneal transendothelial fluid transport mediated by oxidized glutathione and glucose. Exp Eye Res. 1982;35(1):11-19.

13. Reddy VN. Glutathione and its function in the lens--an overview. Exp Eye Res. 1990;50(6):771-778.

14. Ganea E, Harding JJ. Glutathione-related enzymes and the eye. Curr Eye Res. 2006;31(1):1-11.

15. Gekka M, Miyata K, Nagai Y, et al. Corneal epithelial barrier function in diabetic patients. Cornea. 2004;23(1):35-37.
16. Kaiserman I, Kaiserman N, Nakar S, Vinker S. Dry eye in diabetic patients. Am J Ophthalmol. 2005;139(3):498-503.

17. Cousen P, Cackett P, Bennett H, Swa K, Dhillon B. Tear production and corneal sensitivity in diabetes. J Diabetes Complications. 2007;21(6): 371-373.

18. Keoleian GM, Pach JM, Hodge DO, Trocme SD, Bourne WM. Structural and functional studies of the corneal endothelium in diabetes mellitus. Am J Ophthalmol. 1992;113(1):64-70.

19. Larsson LI, Bourne WM, Pach JM, Brubaker RF. Structure and function of the corneal endothelium in diabetes mellitus type I and type II. Arch Ophthalmol. 1996;114(1):9-14.

20. Schultz RO, Matsuda M, Yee RW, Edelhauser HF, Schultz KJ. Corneal endothelial changes in type I and type II diabetes mellitus. Am J Ophthalmol. 1984;98(4):401-410.

21. Lee JS, Oum BS, Choi HY, Lee JE, Cho BM. Differences in corneal thickness and corneal endothelium related to duration in diabetes. Eye (Lond). 2006;20(3):315-318.

22. Bikbova G, Oshitari T, Tawada A, Yamamoto S. Corneal changes in diabetes mellitus. Curr Diabetes Rev. 2012;8(4):294-302.

23. Schultz RO, Van Horn DL, Peters MA, Klewin KM, Schutten WH. Diabetic keratopathy. Trans Am Ophthalmol Soc. 1981;79: 180-199.

24. Sato E, Mori F, Igarashi S, et al. Corneal advanced glycation end products increase in patients with proliferative diabetic retinopathy. Diabetes Care. 2001;24(3):479-482.

25. Knox Cartwright NE, Tyrer JR, Marshall J. Age-related differences in the elasticity of the human cornea. Invest Ophthalmol Vis Sci. 2011;52(7):4324-4329.

26. Rüfer F, Schröder A, Bader C, Erb C. Age-related changes in central and peripheral corneal thickness: determination of normal values with the Orbscan II topography system. Cornea. 2007;26(1):1-5.

27. Roszkowska AM, Colosi P, D’Angelo P, Ferreri G. Age-related modifications of the corneal endothelium in adults. Int Ophthalmol. 2004;25(3):163-166.

28. Redmond P, Burnham JM, Langford MP, Misra RP, Redens TB, Texada DE. Age-related decrease in human corneal $\gamma$-glutamyl transpeptidase activity. Cornea. Epub September 27, 2012.

29. Calderón de la Barca Gázquez JM, Jiménez Alonso J, Barrios L, Jaimez L, Jiménez Murillo L, Jiménez Perepérez JA. Activity of the gamma-glutamyltransferase, leucine aminopeptidase and alkaline phosphatase enzymes in human tear fluid. Enzyme. 1989;41(2): 116-119.

30. Wright JC, Meger GE. A review of the Schirmer test for tear production. Arch Ophthalmol. 1962;67:564-565.

31. Jiménez-Alonso J, Jaimez L, Pérez-Jiménez F, Grilo A, Jiménez-Perepérez JA. Gamma-glutamyltransferase in saliva. Clin Chem. 1982;28(9):1988-1989.

32. Langford MP, Chen D, Neff AG, et al. Intracameral muramyl dipeptide-induced paracellular permeability associated with decreased glutamate transporter and $\gamma$-glutamyl transferase activities. Exp Eye Res. 1999;68(5):591-600.

33. Coupland SE, Penfold PL, Billson FA. Histochemical survey of the anterior segment of the normal human foetal and adult eye. Graefes Arch Clin Exp Ophthalmol. 1993;231(9):533-540.

34. Módis L Jr, Szalai E, Kertész K, Kemény-Beke A, Kettesy B, Berta A. Evaluation of the corneal endothelium in patients with diabetes mellitus type I and II. Histol Histopathol. 2010;25(12):1531-1537.

35. Brom J, Raulf M, Stüning M, et al. Subcellular localization of enzymes involved in leukotriene formation within human polymorphonuclear granulocytes. Immunology. 1984;51(3):571-583.

36. Akinci A, Cetinkaya E, Aycan Z. Dry eye syndrome in diabetic children. Eur J Ophthalmol. 2007;17(6):873-878.

37. Goebbels M. Tear secretion and tear film function in insulin dependent diabetics. Br J Ophthalmol. 2000;84(1):19-21.

38. Gupta A, Heigle T, Pflugfelder SC. Nasolacrimal stimulation of aqueous tear production. Cornea. 1997;16(6):645-648. 
39. Babizhayev MA, Guiotto A, Kasus-Jacobi A. N-Acetylcarnosine and histidyl-hydrazide are potent agents for multitargeted ophthalmic therapy of senile cataracts and diabetic ocular complications. J Drug Target. 2009; 17(1):36-63.
40. Kim J, Kim CS, Kim H, Jeong IH, Sohn E, Kim JS. Protection against advanced glycation end products and oxidative stress during the development of diabetic keratopathy by KIOM-79. J Pharm Pharmacol. 2011;63(4):524-530.

\section{Publish your work in this journal}

Clinical Ophthalmology is an international, peer-reviewed journal covering all subspecialties within ophthalmology. Key topics include: Optometry; Visual science; Pharmacology and drug therapy in eye diseases; Basic Sciences; Primary and Secondary eye care; Patien Safety and Quality of Care Improvements. This journal is indexed on

Submit your manuscript here: http://www.dovepress.com/clinical-ophthalmology-journal

\section{Dovepress}

PubMed Central and CAS, and is the official journal of The Society of Clinical Ophthalmology (SCO). The manuscript management system is completely online and includes a very quick and fair peer-review system, which is all easy to use. Visit http://www.dovepress.com/ testimonials.php to read real quotes from published authors. 